\title{
Confidence intervals for parameters of IWD based on MLE and bootstrap
}

\author{
Mostafa M. MohieEl-Din ${ }^{1}$, Fathy H. Riad ${ }^{2}$, Mohamed A. El-Sayed ${ }^{3,4}$ \\ ${ }^{I}$ Dept. of Mathematics, Faculty of Science, Al-Azhar University, Egypt \\ ${ }^{2}$ Dept. of Mathematics, Faculty of Science, Minia University, Egypt \\ ${ }^{3}$ Dept. of Mathematics, Faculty of Science, In Qena, South Valley University, Egypt \\ ${ }^{4}$ Assistant Professor, Dept. of CS, CIT College, Taif University, KSA \\ E-mail: mas04@fayoum.edu.eg
}

Copyright (1) 2014 Mohamed A. El-Sayed et al. This is an open access article distributed under the Creative Commons Attribution License, which permits unrestricted use, distribution, and reproduction in any medium, provided the original work is properly cited.

\begin{abstract}
In this paper, we will study the joint confidence regions for the parameters of inverse Weibull distribution (IWD) in the point of view of record values. Based on this new censoring scheme, the approximate confidence intervals and percentile bootstrap confidence intervals as well as approximate joint confidence region for the parameters of IWD, are developed. One of the applications of the joint confidence regions of the parameters is to find confidence bounds for the functions of the parameters. Joint confidence regions for the parameters of extreme value distribution are also discussed. In this way we will discuss some numerical examples with real data set and simulated data, to illustrate the proposed method. A simulation study is performed to compare the proposed joint confidence regions.
\end{abstract}

Keywords: IWD, Progressively First-Failure Censored Scheme, MLE Confidence Intervals, Bootstrap Confidence Intervals.

\section{Introduction}

Recently, there are many scenarios in life-testing and reliability experiments whose units are lost or removed from experimentation before failure. The loss may occur un intentionally, or it may have been designed so in the study. The removal of units prior to failure is preplanned in order to provide saving in terms of time and cost associated with testing. There are different types of censored test. Type I and Type II have been investigated extensively in many papers (see, e.g., [1], [4]). A generalization of Type II censoring is progressive Type II censoring. The statistical inference on the parameters of lifetime distribution under progressive censoring has been studied by [5], [7]. In [8] Johnson described a life test in which the experimenter might decide to group the test units into several sets, each as an assembly of test units, and then run all the test units simultaneously until occurrence the first failure in each group. Such a censoring scheme is called first-failure censoring. Jun et al. [9] discussed a sampling plan for a bearing manufacturer. The bearing test engineer decided to save test time by testing 50 bearings in sets of 10 each. The first-failure times from each group were observed. $\mathrm{Wu}$ et al. [10] and $\mathrm{Wu}$ and $\mathrm{Yu}$ [11] obtained maximum likelihood estimates (MLEs), exact confidence intervals and exact confidence regions for the parameters of the Gompertz and Burr type XII distributions based on first-failure-censored sampling, respectively. Also see Lee et al. [12]. Note that a first-failure-censoring scheme is terminated when the first failure in each set is observed. If an experimenter desires to remove some sets of test units before observing the first failures in these sets this life test plan is called a progressive first-failure-censoring scheme which recently introduced by $\mathrm{Wu}$ and Kuş. [13]. in this scheme, first-failure censoring scheme is combined with progressive censoring scheme. For more details on the inverse Weibull distribution, see, for example Johnson et al. [14], Murthy et al. [15] and Mohie El-Din et al [16], [17] .

Suppose that $n$ independent groups with $k$ items within each group are put in a life test. $R_{1}$ groups and the group in which the first failure is observed are randomly removed from the test as soon as the first failure $X_{1 ; m, n, k}^{\mathbf{R}}$ has occurred, $R_{2}$ groups and the group in which the second failure is observed are randomly removed from the test when the second 
failure $X_{2 ; m, n, k}^{\mathbf{R}}$ has occurred, and finally $R_{m}$ groups and the group in which the $m$-th failure is observed are randomly removed from the test as soon as the $m$-th failure $X_{m ; m, n, k}^{\mathbf{R}}$ has occurred. Then $X_{1 ; m, n, k}^{\mathbf{R}}<X_{2 ; m, n, k}^{\mathbf{R}}<\ldots<X_{m ; m, n, k}^{\mathbf{R}}$ are called progressively first-failure censored order statistics with the progressive censored scheme $\mathbf{R}=\left\{R_{1}, R_{2}, \ldots, R_{m}\right\}$ . It is clear that $m$ is number of the first failures $(1<m \leq n)$ and $n=m+\sum_{i=1}^{m} R_{i}$. If the failure times of the ( $\left.n \times k\right)$ items originally in the test are from a continuous population with distribution function $F(x)$ and probability density function $f(x)$, the joint probability density function for $X_{1 ; m, n, k}^{\mathbf{R}}, X_{2 ; m, n, k}^{\mathbf{R}}, \ldots, X_{m ; m, n, k}^{\mathbf{R}}$ is given by

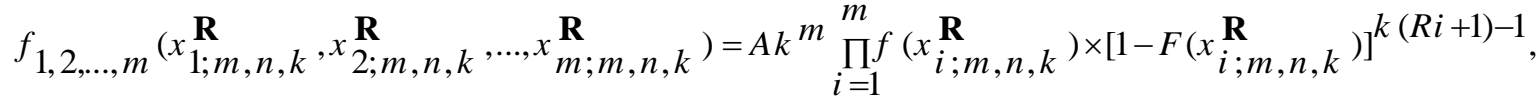

$0<x_{1 ; m, n, k}^{\mathbf{R}}<x_{2 ; m, n, k}^{\mathbf{R}}<\ldots<x_{m ; m, n, k}^{\mathbf{R}}<\infty$,

Where

$A=n\left(n-R_{1}-1\right)\left(n-R_{1}-R_{2}-2\right) \ldots\left(n-R_{1}-R_{2}-\ldots-R_{m-1}-m+1\right)$.

Special cases

It is clear from (1) that the progressive first-failure censored scheme containing the following censoring schemes as special cases:

1) The first-failure censored scheme when $R=\{0,0, \ldots, 0\}$.

2) The progressive Type II censored order statistics if $k=1$.

3) Usually Type II censored order statistics when $k=1$ and $\mathbf{R}=\{0,0, \ldots, n-m\}$.

4) The complete sample case when $k=1$ and $\mathbf{R}=\{0,0, \ldots, 0\}$.

Also, it should be noted that $X_{1 ; m, n, k}^{\mathbf{R}}, X_{2 ; m, n, k}^{\mathbf{R}}, \ldots, X_{m ; m, n, k}^{\mathbf{R}}$ can be viewed as a progressive Type II censored sample from a population with distribution function $1-(1-F(x))^{k}$. For this reason, results for progressive type II censored order statistics can be extend to progressive first-failure censored order statistics easily. Also, the progressive firstfailure-censored plan has advantages in terms of reducing the test time, in which more items are used, but only $m$ of $n \times k$ items are failures.

In many practical situations, it is often known a priori that the hazard rate cannot be monotone. It may happen that the course of a disease is such that the mortality reaches a peak after some finite period, and then declines slowly. Bennett [18] analyzed the data from the Veterans Administration lung cancer trial presented by Prentice [19] and showed that the empirical failure rates for both low and high performance status groups were unimodal in nature. It is important to analyze such data sets with appropriate models. If the empirical studies indicate that the hazard function might be unimodal, then the IWD may be an appropriate model.

The probability density function $(P D F)$ and the cumulative distribution $(C D F)$ of the IWD as follows:

$f(x)=\alpha \beta x^{-\beta-1} e^{-\alpha x^{-\beta}}, \quad x>0, \alpha, \beta>0$,

And

$F(x)=e^{-\alpha x^{-\beta}}, \quad x>0, \alpha, \beta>0$,

As in the Weibull distribution, the shape parameter $\beta$ governs the shape of the $p d f$, the hazard function and the general properties of the IWD. PDF and HF can be unimodal or decreasing depending on the choice of the shape parameter. In this respect the behavior of IWD and the log-normal distribution is quite similar.

The rest of the paper is organized as follows. In section 2, we provide The ML point and interval estimates of the parameters as well as approximate joint confidence region for the parameters of IWD. The parametric bootstrap confidence intervals of parameters are proposed in this section 3. Monte Carlo simulation results are presented in section 4. Data analysis is provided in section 5, and finally we conclude the paper in section 6.

\section{Maximum likelihood estimation}

To determine the point estimation, Let $\underline{X}=\left(X_{1 ; m, n, k}^{\mathbf{R}}, X_{2 ; m, n, k}^{\mathbf{R}}, \ldots, X_{m ; m, n, k}^{\mathbf{R}}\right)$ be the progressive first-failure censored order statistics from inverse Weibull distribution with censored scheme R. From (1), (2) and (3), the likelihood function is given by

$\ell(\alpha, \beta \mid \underline{X})=m \log \alpha+m \log \beta-(\beta+1) \sum_{i=1}^{m} \log x_{i}-\alpha \sum_{i=1}^{m} x_{i}^{-\beta}+\sum_{i=1}^{m}\left(k\left(R_{i}+1\right)-1\right) \log \left(1-F\left(x_{i}\right)\right)$

Calculating the first partial derivatives of (5) with respect to $\alpha$ and $\beta$ and equating to zero, we obtain the likelihood equations 
$\frac{\partial \ell(\alpha, \beta \mid \underline{X})}{\partial \alpha}=m / \beta-\sum_{i=1}^{m} x_{i}^{-\beta}+\sum_{i=1}^{m}\left(k R_{i}+k-1\right) x_{i}^{-\beta} \psi\left(x_{i}\right)=0$,

where, $\psi\left(x_{i}\right)=F\left(x_{i}\right) /\left(1-F\left(x_{i}\right)\right)$ and

$\frac{\partial \ell(\alpha, \beta \mid \underline{X})}{\partial \beta}=m / \beta-\sum_{i=1}^{m} \log x_{i}+\alpha \sum_{i=1}^{m} x_{i}^{-\beta} \log x_{i}-\alpha \sum_{i=1}^{m}\left(k R_{i}+k-1\right) x_{i}^{-\beta} \log x_{i} \psi\left(x_{i}\right)=0$

The maximum likelihood (ML) estimates $\hat{\alpha}$ and $\hat{\beta}$ of the parameters can be obtained by solving the two nonlinear Eqs. (6) and (7), some numerical methods such as Newton's method can be employed.

\section{Approximate interval estimation}

The asymptotic variances and covariances of the MLE for parameters $\beta$ and $\alpha$ are given by elements of the inverse of the Fisher information matrix

$\mathbf{I}_{i j}=E\left[-\frac{\partial^{2} L}{\partial \beta \partial \alpha}\right] ; i, j=1,2$.

Unfortunately, the exact mathematical expressions for the above expectations are very difficult to obtain. Therefore, we give the approximate (observed) asymptotic variance-covariance matrix for the MLE, which is obtained by dropping the expectation operator $E$

$I_{0}^{-1}(\hat{\alpha}, \hat{\beta})=\left[\begin{array}{cc}-\frac{\partial^{2} L}{\partial \alpha^{2}} & -\frac{\partial^{2} L}{\partial \alpha \partial \beta} \\ -\frac{\partial^{2} L}{\partial \beta \partial \alpha} & -\frac{\partial^{2} L}{\partial \beta^{2}}\end{array}\right]_{(\hat{\alpha}, \hat{\beta})}^{-1}=\left[\begin{array}{lr}\operatorname{var}(\hat{\alpha}) & \operatorname{Cov}(\hat{\alpha}, \hat{\beta}) \\ \operatorname{Cov}(\hat{\beta}, \hat{\alpha}) & \operatorname{var}(\hat{\beta})\end{array}\right]$

with

$\frac{\partial^{2} L}{\partial \beta^{2}}=-\frac{m}{\beta^{2}}+\alpha \sum_{i=1}^{m} x_{i}^{-\beta}\left(\log x_{i}\right)^{2}-\alpha \sum_{i=1}^{m}\left(k R_{i}+k-1\right) x_{i}^{-\beta}\left(\log x_{i}\right)^{2}\left[\alpha x_{i}^{-\beta} e^{\alpha x_{i}^{-\beta}}-e^{\alpha x_{i}^{-\beta}}+1\right]\left(e^{\alpha x_{i}^{-\beta}}-1\right)^{-2}$,

$\frac{\partial^{2} L}{\partial \alpha^{2}}=-\frac{m}{\alpha^{2}}-\sum_{i=1}^{m}\left(k R_{i}+k-1\right) e^{\alpha x_{i}^{-\beta}} x_{i}^{-2 \beta}\left(e^{\alpha x_{i}^{-\beta}}-1\right)^{-2}$

and

$\frac{\partial^{2} L}{\partial \beta \partial \alpha} \equiv \frac{\partial^{2} L}{\partial \alpha \partial \beta}=\sum_{i=1}^{m} x_{i}^{-\beta} \log x_{i}+\sum_{i=1}^{m}\left(k R_{i}+k-1\right) x_{i}^{-\beta} \log x_{i}\left[\left(\alpha x_{i}^{-\beta}-1\right) e^{\alpha x_{i}^{-\beta}}-1\right]\left(e^{\alpha x_{i}^{-\beta}}-1\right)^{-2}$

Approximate confidence intervals for $\beta$ and $\alpha$ can be found by taking $(\hat{\beta}, \hat{\alpha})$ to be bivariantely normally distributed with mean $(\beta, \alpha)$ and covariance matrix $I_{0}^{-1}(\hat{\beta}, \hat{\alpha})$ i.e. $(\hat{\beta}, \hat{\alpha}) \sim N\left((\beta, \alpha), I_{0}^{-1}(\hat{\beta}, \hat{\alpha})\right)$.

Thus, the $(1-\gamma) 100 \%$ confidence intervals for parameters $\beta$, and $\alpha$ become

$\hat{\alpha} \pm Z_{\gamma / 2} \sqrt{\operatorname{var}(\hat{\alpha})}$ and $\hat{\beta} \pm Z_{\gamma / 2} \sqrt{\operatorname{var(\hat {\beta })}}$

where $Z_{\gamma / 2}$ is the percentile of the standard normal distribution with right-tail probability.

The approximate confidence region for $(\alpha, \beta)$ the normal approximation for $(\hat{\alpha}, \hat{\beta})$ implies that

$[\hat{\alpha}-\alpha \hat{\beta}-\beta] I_{0}^{-1}(\hat{\alpha}, \hat{\beta})[\hat{\alpha}-\alpha \hat{\beta}-\beta]^{t}$

is asymptotically of chi-squared form with two degrees of freedom. Let

$Q=\left\{(\alpha, \beta):[\hat{\alpha}-\alpha \hat{\beta}-\beta] I_{0}^{-1}(\hat{\alpha}, \hat{\beta})[\hat{\alpha}-\alpha \hat{\beta}-\beta]^{t} \leq \chi_{2(\gamma)}^{2}\right\}$

where $\chi_{2(\gamma)}^{2}$ is the percentile of the chi-squared distribution with right-tail probability and two degrees of freedom. This can be used to obtain the $100(1-\gamma) \%$ approximate confidence region for $(\alpha, \beta)$.

\section{Bootstrap confidence Intervals}

The bootstrap is a resampling method for statistical inference. It is commonly used to estimate confidence intervals, but it can also be used to estimate bias and variance of an estimator or calibrate hypothesis tests. More survey of the nonparametric and parametric bootstrap methods (Davison and Hinkley, [20]), Efron [21]. In this Section, we used the parametric bootstrap percentile method suggested by Efron and Tibshirani [22] to construct confidence intervals for the parameters. The following steps are followed to obtain progressive first failure censoring bootstrap sample from IWD with parameters $\alpha$ and $\beta$ based on simulated progressively first-failure censored data set.

Algorithm 2.1: 
Step1: From an original data set $x \equiv\left(x_{1 ; m, n, k}^{\mathbf{R}}, x_{2 ; m, n, k}^{\mathbf{R}}, \ldots, x_{m ; m, n, k}^{\mathbf{R}}\right)$, compute the ML estimates of the parameters $\alpha$ and $\beta$ from equations (8) and (9) .

Step2: Use $\hat{\alpha}$ and $\hat{\beta}$ to generate a bootstrap sample $\underline{x}^{*}$ with the same values of $R_{i},(i=1,2, \ldots, m)$ using the algorithm of Balakrishnan and Sandhu [7] .

Step3: As in step 1 based on $x^{*}$ compute the bootstrap sample estimates of $\alpha$ and $\beta$ say $\hat{\alpha}^{*}$ and $\hat{\beta}^{*}$.

Step4: Repeat steps 2 and $3 N$ times representing $N$ bootstrap ML estimates of $\alpha$ and $\beta$ based on $N$ different bootstrap samples.

Step5: Arrange a $\hat{\alpha}^{* i}$ and $\hat{\beta}^{* i}$ in an ascending order to obtain bootstrap sample $\left(\phi_{t}^{(1)}, \phi_{t}^{(2)}, \ldots, \phi_{t}^{(N)}\right), t=1,2$ where ( $\left(\phi_{1}=\hat{\alpha}^{*}, \phi_{2}=\hat{\beta}^{*}\right)$. Let $\mathrm{G}(\mathrm{z})=P\left(\phi_{t} \leq z\right)$ be cumulative distribution function of $\phi_{t}$. Define $\phi_{t b o o t}=G^{-1}(z)$ for given $z$. The approximate bootstrap $100(1-\gamma) \%$ confidence interval of $\phi_{t}$ given by:

$\left[\phi_{t b o o t}\left(\frac{\gamma}{2}\right), \phi_{t b o o t}\left(1-\frac{\gamma}{2}\right)\right]$.

\section{Simulation study}

To study the performance of our approach, we simulated 1000 progressively first-failure censored samples from a IWD with the values of parameters $(\alpha, \beta)=(1.5,0.5)$ and different combinations of $n, m, k$ and censoring schemes $\mathbf{R}$. The samples were simulated by using the algorithm described in Balakrishnan and Sandhu [7]. First, we study the properties of the MLEs for different choices of $n, m, k$, and progressive censoring schemes $\mathbf{R}$.

Table 1 provide the Average values and mean squared errors (MSEs) of the ML and bootstrap estimates. In our study we have used three different censoring schemes (CS), namely:

CS 1: for $R_{1}=n-m, \quad R_{i}=0$ for $i \neq m$.

CS 2: for $R_{m}=n-m, \quad R_{i}=0$ for $i \neq 1$.

CS 3: for $R_{(m+1) / 2}=n-m, R_{i}=0$ for $i \neq(m+1) / 2$; if $m$ odd,

and $R_{m / 2}=n-m, R_{i}=0$ for $i \neq m / 2$; if $m$ even.

Table 1: Average values and MSEs of the estimates of $\alpha$ and $\beta$ with $(\alpha=1, \beta=2)$.

\begin{tabular}{|c|c|c|c|c|c|c|c|}
\hline \multirow{2}{*}{$k, n, m$} & \multirow{2}{*}{ Method } & \multicolumn{2}{|c|}{ Scheme CS 1} & \multicolumn{2}{|c|}{ Scheme CS 2} & \multicolumn{2}{|c|}{ Scheme CS 3} \\
\hline & & $\hat{\alpha}(\mathrm{MSE})$ & $\hat{\beta}(\mathrm{MSE})$ & $\hat{\alpha}(\mathrm{MSE})$ & $\hat{\beta}(\mathrm{MSE})$ & $\hat{\alpha}(\mathrm{MSE})$ & $\hat{\beta}(\mathrm{MSE})$ \\
\hline \multirow{2}{*}{$1,20,10$} & MLE & $1.0984(0.4479)$ & $2.1765(0.7188)$ & $1.0307(0.3075)$ & $2.3306(0.7266)$ & $0.9727(0.3061)$ & $2.3490(0.7469)$ \\
\hline & B-strap & $1.1344(0.5158)$ & $2.4568(0.9047)$ & $1.0503(0.3488)$ & 2.7268 ( 1.0469$)$ & $0.9766(0.3449)$ & $2.7280(1.0529)$ \\
\hline \multirow{2}{*}{$1,20,15$} & MLE & $1.0427(0.3136)$ & $2.2288(0.5425)$ & $1.0304(0.2991)$ & $2.2008(0.4907)$ & $0.9983(0.2829)$ & $2.2013(0.4951)$ \\
\hline & B-strap & $1.0909(0.3600)$ & $2.4451(0.7076)$ & $1.0762(0.3504)$ & $2.4287(0.6576)$ & $1.0339(0.3248)$ & $2.4348(0.6666)$ \\
\hline \multirow{2}{*}{$1,50,20$} & MLE & $0.9953(0.2031)$ & $2.0768(0.3465)$ & $1.0106(0.2025)$ & $2.1617(0.4066)$ & $0.9721(0.1866)$ & $2.1417(0.3844)$ \\
\hline & B-strap & $0.9987(0.2096)$ & $2.1900(0.4069)$ & $1.0011(0.2098)$ & $2.3342(0.5263)$ & $0.9582(0.1928)$ & $2.2992(0.4900)$ \\
\hline \multirow{2}{*}{$1,50,30$} & MLE & $1.0454(0.2178)$ & $2.0469(0.2863)$ & $0.9959(0.1672)$ & $2.0700(0.3177)$ & $1.0020(0.1689)$ & $2.0973(0.3217)$ \\
\hline & B-strap & $1.0568(0.2288)$ & $2.1304(0.3232)$ & $1.0039(0.1755)$ & $2.1714(0.3673)$ & $1.0042(0.1771)$ & $2.2000(0.3816)$ \\
\hline \multirow{2}{*}{$3,20,10$} & MLE & $0.9380(0.2732)$ & $2.2081(0.5207)$ & $0.9527(0.2643)$ & $2.3104(0.6760)$ & $0.9624(0.2736)$ & $2.2822(0.7275)$ \\
\hline & B-strap & $0.8977(0.2850)$ & $2.4546(0.6975)$ & $0.8754(0.2875)$ & $2.7182(1.0019)$ & $0.8985(0.2845)$ & $2.6120(0.9700)$ \\
\hline \multirow{2}{*}{$3,20,15$} & MLE & $0.9641(0.2248)$ & $2.2044(0.5129)$ & $0.9496(0.2140)$ & $2.2543(0.5231)$ & $0.9387(0.2145)$ & $2.2078(0.5169)$ \\
\hline & B-strap & $0.9429(0.2307)$ & $2.3898(0.6481)$ & $0.9192(0.2209)$ & $2.4770(0.6918)$ & $0.9096(0.2223)$ & $2.4193(0.6748)$ \\
\hline \multirow{2}{*}{$3,50,20$} & MLE & $0.9721(0.1865)$ & $2.0909(0.3177)$ & $0.9506(0.1938)$ & $2.1899(0.4587)$ & $0.9652(0.1889)$ & $2.0927(0.3768)$ \\
\hline & B-strap & $0.9526(0.1862)$ & $2.1879(0.3661)$ & $0.9038(0.2100)$ & $2.3735(0.5890)$ & $0.9312(0.1972)$ & $2.2216(0.4500)$ \\
\hline \multirow{2}{*}{$3,50,30$} & MLE & $0.9845(0.1575)$ & $2.1094(0.3004)$ & $0.9823(0.1552)$ & $2.1000(0.3227)$ & $0.9616(0.1612)$ & $2.1359(0.3493)$ \\
\hline & B-strap & $0.9698(0.1602)$ & $2.1895(0.3482)$ & $0.9609(0.1593)$ & $2.2059(0.3838)$ & $0.9427(0.1672)$ & $2.2305(0.4112)$ \\
\hline \multirow{2}{*}{$5,20,10$} & MLE & $0.9566(0.2913)$ & $2.2420(0.6659)$ & $0.8966(0.2929)$ & $2.3376(0.7364)$ & $0.9082(0.2710)$ & $2.3152(0.5689)$ \\
\hline & B-strap & $0.8985(0.3007)$ & $2.4933(0.8523)$ & $0.7987(0.3301)$ & $2.7420(1.0523)$ & $0.8302(0.2990)$ & $2.6408(0.8373)$ \\
\hline \multirow{2}{*}{$5,20,15$} & MLE & $0.9121(0.2244)$ & $2.2439(0.5342)$ & $0.9390(0.2059)$ & $2.1877(0.4680)$ & $0.9917(0.2249)$ & $2.0813(0.4465)$ \\
\hline & B-strap & $0.8730(0.2411)$ & $2.4270(0.6729)$ & $0.8874(0.2253)$ & $2.4135(0.6289)$ & $0.9437(0.2303)$ & $2.2749(0.5538)$ \\
\hline \multirow{2}{*}{$5,50,20$} & MLE & $0.9571(0.1945)$ & $2.1072(0.3443)$ & $0.9295(0.2036)$ & $2.1715(0.3828)$ & $0.9550(0.1982)$ & $2.1332(0.3638)$ \\
\hline & B-strap & $0.9288(0.2013)$ & $2.2057(0.4024)$ & $0.8714(0.2263)$ & $2.3566(0.5148)$ & $0.9124(0.2078)$ & $2.2654(0.4491)$ \\
\hline \multirow{2}{*}{$5,50,30$} & MLE & $0.9999(0.1218)$ & $2.0470(0.2454)$ & $0.9685(0.1524)$ & $2.1228(0.3218)$ & $0.9867(0.1501)$ & $2.0681(0.2837)$ \\
\hline & B-strap & $0.9806(0.1236)$ & $2.1212(0.2773)$ & $0.9384(0.1593)$ & $2.2262(0.3861)$ & $0.9597(0.1533)$ & $2.1599(0.3313)$ \\
\hline
\end{tabular}


Table 2: Coverage probability and average length for the approximate MLE and bootstrap $(\alpha=1, \beta=2)$.

\begin{tabular}{|c|c|c|c|c|c|c|c|}
\hline \multirow{2}{*}{$k, n, m$} & \multirow{2}{*}{ Method } & \multicolumn{2}{|c|}{ Scheme CS 1 - coverage(length) } & \multicolumn{2}{|c|}{ Scheme CS 2 - coverage(length) } & \multicolumn{2}{|c|}{ Scheme CS 3- coverage(length) } \\
\hline & & $\hat{\alpha}$ & $\hat{\beta}$ & $\hat{\alpha}$ & $\hat{\beta}$ & $\hat{\alpha}$ & $\hat{\beta}$ \\
\hline \multirow{2}{*}{$1,20,10$} & MLE & $0.95(1.3271)$ & $0.89(2.0117)$ & $0.92(1.0449)$ & $0.96(2.2000)$ & $0.91(1.0868)$ & $0.95(2.2617)$ \\
\hline & B-strap & $0.93(1.5436)$ & $0.90(2.6531)$ & $0.92(1.2957)$ & $0.81(3.1437)$ & $0.90(1.2976)$ & $0.82(3.1428)$ \\
\hline \multirow{2}{*}{$1,20,15$} & MLE & $0.91(1.0814)$ & $0.93(1.7340)$ & $0.95(0.9764)$ & $0.96(1.7097)$ & $0.91(0.9760)$ & $0.98(1.7607)$ \\
\hline & B-strap & $0.93(1.2983)$ & $0.89(2.1449)$ & $0.92(1.1795)$ & $0.88(2.1141)$ & $0.93(1.1674)$ & $0.89(2.1811)$ \\
\hline \multirow{2}{*}{$1,50,20$} & MLE & $0.98(0.8784)$ & $0.98(1.3149)$ & $0.96(0.6955)$ & $0.94(1.4406)$ & $0.96(0.7494)$ & $0.97(1.4456)$ \\
\hline & B-strap & $1.00(0.9376)$ & $0.93(1.4968)$ & $0.95(0.7540)$ & $0.88(1.7379)$ & $0.97(0.7896)$ & $0.86(1.7089)$ \\
\hline \multirow{2}{*}{$1,50,30$} & MLE & $0.94(0.7594)$ & $0.97(1.0930)$ & $0.94(0.6160)$ & $0.92(1.1166)$ & $0.96(0.6513)$ & $0.95(1.1649)$ \\
\hline & B-strap & $0.92(0.8140)$ & $0.94(1.2359)$ & $0.95(0.6589)$ & $0.91(1.2509)$ & $0.93(0.6960)$ & $0.90(1.2954)$ \\
\hline \multirow{2}{*}{$3,20,10$} & MLE & $0.90(0.9851)$ & $0.97(1.8423)$ & $0.95(0.9705)$ & $0.95(2.1747)$ & $0.90(0.9928)$ & $0.95(2.0262)$ \\
\hline & B-strap & $0.87(1.0156)$ & $0.84(2.38$ & $0.88(1.0194)$ & $0.82(3.1101)$ & $0.91(1.0297)$ & $0.88(2.7485)$ \\
\hline \multirow{2}{*}{$3,20,15$} & MLE & $0.95(0.8112)$ & $0.91(1.5804)$ & $0.92(0.7745)$ & $0.95(1.6875)$ & $0.90(0.7948)$ & $0.92(1.6423)$ \\
\hline & B-strap & $0.96(0.8460)$ & $0.87(1.93$ & $0.91(0.8119)$ & $0.78(2.14$ & $0.90(0.8229)$ & $0.87(2.0389)$ \\
\hline \multirow{2}{*}{$3,50,20$} & MLE & $0.91(0.7052)$ & $0.96(1.1970)$ & $0.90(0.7181)$ & $0.94(1.4809)$ & $0.93(0.7249)$ & $0.95(1.3037)$ \\
\hline & B-strap & $0.92(0.7$ & 1.3 & 0.89 ( 0 . & $0.86(1.7$ & $0.90(0$. & $0.92(1.5314)$ \\
\hline \multirow{2}{*}{$3,50,30$} & MLE & $0.94(0.5794)$ & $0.95(1.0429)$ & $0.93(0.5595)$ & $0.93(1.1265)$ & $0.92(0.5728)$ & $0.93(1.1047)$ \\
\hline & B-strap & 0.96 ( 0.6 & $0.86(1.1$ & $0.93(0.5840)$ & $0.92(1.2906)$ & $0.88(0.5879)$ & $0.87(1.2273)$ \\
\hline \multirow{2}{*}{$5,20,10$} & MLE & $0.86(0.9$ & $0.89(1.8124)$ & $0.89(1.0233)$ & $0.95(2.2245)$ & $0.90(1.0010)$ & $0.98(2.0114)$ \\
\hline & B-strap & $0.84(0.9$ & $0.84(2.3541)$ & $0.84(1.0240)$ & $0.80(3.0246)$ & $0.86(1.0116)$ & $0.84(2.7656)$ \\
\hline \multirow{2}{*}{$5,20,15$} & MLE & $0.90(0.7745)$ & $0.95(1.5710)$ & $0.95(0.7975)$ & $0.97(1.6394)$ & $0.92(0.8152)$ & $0.93(1.5144)$ \\
\hline & B-strap & $0.87(0.7958)$ & $0.83(1.8788)$ & $0.90(0.8188)$ & $0.85(2.0672)$ & $0.92(0.8423)$ & $0.90(1.8755)$ \\
\hline \multirow{2}{*}{$5,50,20$} & MLE & $0.93(0.6833)$ & $0.94(1.1801)$ & $0.91(0.7812)$ & $0.99(1.4868)$ & $0.91(0.7428)$ & $0.97(1.2972)$ \\
\hline & B-strap & $0.92(0.6917)$ & $0.90(1.3182)$ & $0.86(0.7980)$ & $0.92(1.8092)$ & $0.89(0.7628)$ & $0.92(1.5188)$ \\
\hline \multirow{2}{*}{$5,50,30$} & MLE & $0.98(0.569)$ & $0.97(0.9911)$ & $0.95(0.5925)$ & $0.96(1.1460)$ & $0.95(0.5933)$ & $0.93(1.0558)$ \\
\hline & B-strap & $0.98(0.5852)$ & $0.96(1.0954)$ & $0.91(0.6036)$ & $0.89(1.2904)$ & $0.94(0.6131)$ & $0.94(1.1839)$ \\
\hline
\end{tabular}

It is important to examine how well our proposed method works for constructing confidence intervals and regions. We will compare the approximate confidence intervals or regions on the basis of asymptotic properties of the MLEs with the bootstrap confidence interval discussed in Section 4 in terms of coverage probabilities. The coverage probability average length is the probability that the interval or region contains the true parameters. The simulation results of the coverage probabilities and average length are summarized in Table 2.

\section{Illustrative example}

For illustrative purpose, we consider a progressive first-failure censored sample were generated from IWD with parameters: $\alpha=2, \beta=1$, using the algorithm proposed by Balakrishnan and Sandhu [7]. The data consisting of 150 observations as a lifetime data, were randomly grouped into $n=30$ sets, with 5 observations in each. The progressive first-failure censored sample with the corresponding censoring scheme are given in Table 3.

\begin{tabular}{ccccccccccccc}
\multicolumn{10}{c}{ Table 3: Simulated progressive first-failure censored sample } \\
\hline$i$ & 1 & 2 & 3 & 4 & 5 & 6 & 7 & 8 & 9 & 10 \\
\hline$R_{i}$ & 5 & 0 & 3 & 3 & 0 & 1 & 0 & 3 & 0 & 5 \\
$X_{i ; 8,26,5}^{R}$ & 0.3153 & 0.3703 & 0.5582 & 0.6912 & 0.7437 & 0.7661 & 0.7882 & 0.8386 & 0.99145 & 1.0409 \\
\hline
\end{tabular}

Based on progressive first-failure sampling given in Table 3. Under the data given in Table 3 we compute MLEs and bootstrap estimates of $\alpha$ and $\beta$ results are given in Table 4 .

Table 4: Results obtained by MLE, Bootstrap method, of $\alpha$ and $\beta$.

\begin{tabular}{cccc}
\hline Parameter & True value & $(.)_{\text {MLE }}$ & $(.)_{\text {Boot }}$ \\
\hline$\alpha$ & 2.0 & 2.09252 & 1.9691 \\
$\beta$ & 1.0 & 0.957363 & 1.0983 \\
\hline
\end{tabular}

Table 5 gives the $95 \%$, approximate $M L E$ confidence intervals and percentile bootstrap confidence interval.

Table 5: Two-sided 95\% confidence intervals of $\alpha$ and $\beta$.

\begin{tabular}{ccccc}
\hline Methods of Estimate & $95 \%$ C.I of $\alpha$ & Length & 95\% C.I of $\beta$ & Length \\
\hline MLE & $(1.50641,2.67864)$ & 1.18223 & $(0.53840,1.37633)$ & 0.83793 \\
Bootstrap & $(1.3897,2.6126)$ & 1.2229 & $(0.7268,1.849)$ & 1.1222 \\
\hline
\end{tabular}




\section{Conclusion}

Censoring is a common phenomenon in life-testing and reliability studies. The subject of progressive censoring has received considerable attention in the past few years, due in part to the availability of high speed computing resources, which makes it both a feasible topic for simulation studies for researchers and a feasible method of gathering lifetime data for practitioners.

In this article, we have considered the maximum likelihood and bootstrap for the parameters of the IWD using progressive first-failure censored scheme. Also, we develop an approximate confidence intervals and an approximate joint confidence region for the parameters of the IWD. A simulation study was conducted to examine and compare the performance of the proposed methods

1) From Tables 1-2, as the effective sample proportion $m / n$ increases, the $M S E s$ of the estimators, reduce significantly. For fixed $n, m$ and $k$, we can determine the censoring scheme which is most efficient; for example, from tables, we observe that the censoring scheme $C S 2$, corresponding to the case of withdrawal in the first stage of the test, seems to provide the smallest $M S E$ for the estimate of the parameters.

2) The MSEs for all estimates based on the progressive first-failure censoring scheme with $k=3$ and $k=5$ are similar to those for progressive Type II censoring with $k=1$.

3) From Tables 5 the coverage probabilities of the approximate confidence intervals for $\alpha$ and $\beta$, and the approximate confidence region for $(\hat{\alpha}, \hat{\beta})$ are almost all close to the desired level of 0.95 .

\section{References}

[1] N. R. Mann,'Best linear invariant estimation for Weibull parameters under progressive censoring", Technometrics, Vol. 13, (1971). pp. 521533

[2] J. F. Lawless,"Statistical Models and Methods for Lifetime Data", John Wiley \& Sons, New York, NY, USA (1982).

[3] W. Q. Meeker, and L. A. Escobar,"Statistical Methods for Reliability Data”, John Wiley \& Sons, New York, NY, USA (1998).

[4] S. K. Tse, and H. K.Yuen,"Expected experiment times for the Wiebull distribution under progressive censoring with random removals", journal of Applied Statistics, Vol. 25, (1998), pp. 75-83.

[5] A. C. Cohen,'Progressively censored samples in life testing", Technometrics, Vol. 5, (1963), pp. $327-339$.

[6] R. Viveros, and N. Balakrishnan,"Interval estimation of parameters of life from progressively censored data", Technometrics, Vol. 36, No. 1, (1994), pp. 84-91.

[7] N. Balakrishnan, and R. A. Sandhu'Best linear unbiased and maximum likelihood estimation for exponential distributions under general progressive type-II censored samples", Sankhya. Series B, Vol. 58, No. 1, (1996), pp. 1-9.

[8] N. L. Johnson, S. Kotz, N. Balakrishnan,"Continuous Univariate Distributions”, Vol. 2. 2nd Ed. New York: John Wiley \& Sons (1995)

[9] C.-H. Jun, S. Balamurali, and S.-H. Lee,"Variables sampling plans for Weibull distributed lifetimes under sudden death testing". IEEE Transactions on Reliability Vol.55, (2006) pp. 53-58.

[10] J. W. Wu, H. Y. Yu , "Statistical inference about the shape parameter of the Burr type XII distribution under the failure-censored sampling plan", Applied Mathematics and Computation Vol. 163, (2005). Pp.443-482.

[11] W.-C. Lee, J.-W. Wu, H.-Y. Yu," Statistical inference about the shape parameter of the bathtub-shaped distribution under the failure-censored sampling plan" International Journal of Information and Management Sciences Vol. 18, (2007). pp. 157-172.

[12] S. J. Wu, C. Kuş, ,On estimation based on progressive first-failure-censored sampling”. Computational Statistics and Data Analysis Vol. 53 (2009). pp. 3659- 3670.

[13] A.O. Langlands, S.J. Pocock, G.R. Kerr, and S.M. Gore,’Long term survival of patients with breast cancer: a study of curability of the disease", British Medical Journal, (1979), pp. 247-125.

[14] N. L. Johnson, S. Kotz, and N. Balakrishnan, Continuous Univariate Distributions. Vol. 2, second edition. John Wiley \& Sons New York, (1995).

[15] D. N. P. Murthy, M. Xie, and R. Jiang, Weibull Model. John Wiley \& Sons, New York, (2004).

[16] M. M, Mohie El-Din,,and F. H. Riad, Estimation and Prediction for the Inverse Weibull Distribution Based on Records, Journal of Advanced Research in Statistics and Probability (JARSP), 3(2), 20 - 27, (2011).

[17] Mostafa. M. MohieEl-Din, Fathy H. Riad and Mohamed A. El-Sayed, Parameters Estimation Based On Progressively Censored Data From Inverse Weibull Distribution, American Journal of Theoretical and Applied Statistics (AJTAS), 2(6): 149-153, 2013.

[18] S. Bennette,'Log-logistic regression models for survival data", Applied Statistics, Vol. 32, (1983), pp. $165-171$.

[19] R.L. Prentice,"Exponential survivals with censoring and explanatory variables", Biometrika, Vol. 60, (1973), pp. 279-288.

[20] A. C. Davison and D. V. Hinkley "Bootstrap Methods and their Applications", $2^{\text {nd }}$ Cambridge University Press, Cambridge United Kingdom, (1997).

[21] B. Efron, "The jackknife, the bootstrap and other resampling plans", CBMS-NSF Regional Conference Series in Applied Mathematics, 38, SIAM, Philadelphia, PA. (1982).

[22] B. Efron, and R. J. Tibshirani, "Bootstrap method for standard errors, confidence intervals and other measures of statistical accuracy", Statistical Science, Vol.1, (1986), pp. 54-75. 